\title{
High Fidelity Single Qubit Operations Using Pulsed Electron Paramagnetic Resonance
}

\author{
John J.L. Morton, ${ }^{1, *}$ Alexei M. Tyryshkin, ${ }^{2}$ Arzhang Ardavan, ${ }^{3}$ Kyriakos Porfyrakis, ${ }^{1}$ \\ S. A. Lyon, ${ }^{2}$ and G. Andrew D. Briggs ${ }^{1}$ \\ ${ }^{1}$ Department of Materials, Oxford University, Oxford OX1 3PH, United Kingdom \\ ${ }^{2}$ Department of Electrical Engineering, Princeton University, Princeton, New Jersey 08544, USA \\ ${ }^{3}$ Clarendon Laboratory, Department of Physics, Oxford University, Oxford OXI 3PU, United Kingdom
}

(Received 4 May 2005; published 8 November 2005)

\begin{abstract}
Systematic errors in spin rotation operations using simple rf pulses place severe limitations on the usefulness of the pulsed magnetic resonance methods in quantum computing applications. In particular, the fidelity of quantum logic operations performed on electron spin qubits falls well below the threshold for the application of quantum algorithms. Using three independent techniques, we demonstrate the use of composite pulses to improve this fidelity by several orders of magnitude. The observed high-fidelity operations are limited by pulse phase errors, but nevertheless fall within the limits required for the application of quantum error correction.
\end{abstract}

Pulsed magnetic resonance, which provides a way of manipulating quantum systems such as nuclear and electron spins, has proved to be a powerful tool in the development of quantum computation. This was borne by the early success of nuclear magnetic resonance (NMR) quantum computers, which have performed the largest-scale quantum computations to date [1,2]. Always-on dipolar or exchange interactions are exploited to yield multiqubit gates, while single-qubit operations are performed using classical rf pulses. The scalability limitations surrounding NMR implementations [3] that arise from the small nuclear Zeeman energy can be overcome by turning to electron paramagnetic resonance (EPR), analogous in many ways to NMR but with the advantage that pure states are experimentally accessible.

These strengths have prompted many EPR-based solid state quantum information processing proposals [4-7]. The merit of such schemes is often argued on the basis of the decoherence time $T_{2}$. However, the fidelity with which operations can be performed also imposes severe limitations on the viability of such proposals. We recently developed a methodology for characterizing systematic errors in pulsed EPR, and used it to measure typical errors in a commercial EPR spectrometer [8]. The most significant error present in a single-qubit operation is in the rotation angle, arising from spatial inhomogeneity in the pulsed rf field. This systematic error in the rotation angle is likely to persist even in the case of single-molecule EPR experiments, caused by miscalibrated control equipment. Fortunately, a number of approaches to tackling different classes of systematic errors have been developed in the art of NMR, employing composite rotation sequences $[9,10]$. A small subset of such approaches correct rotation operators rather than final states and are therefore successful regardless of the initial spin state. These approaches are applicable to quantum computing [11-14]. Of these, the BB1 sequence [11] exploits the precision in pulse phase control to correct for systematic errors in rotation angle and is therefore ideal for our purposes.

High-fidelity pulses are also beneficial to more traditional EPR characterization techniques such as correlation spectroscopy (e.g., 2D-HYSCORE [15]) by eliminating or suppressing any spurious cross-peak signals and thus simplifying the spectral analysis.

In this Letter, we show how composite pulses can be applied in pulsed EPR to perform high-fidelity operations on electron spins. We use these to demonstrate nondecaying Rabi oscillations, and provide further evidence using an error-sensitive electron spin echo envelope modulation (ESEEM) effect [16]. Finally, we use the error-measuring sequences described in [8] to estimate the residual error in the composite pulse. We find that, in current EPR spectrometers, the chief limitation lies in the ability to accurately set the pulse phase, which restricts the effectiveness of the composite pulse.

The paramagnetic species used here to perform error measurements in pulsed EPR is $i-\mathrm{NC}_{60}$ (also known as $\mathrm{N} @ \mathrm{C}_{60}$ ), consisting of an isolated nitrogen atom in the ${ }^{4} S_{3 / 2}$ electronic state incarcerated by a $\mathrm{C}_{60}$ fullerene cage. It is an ideal system for these measurements because of its extremely narrow EPR linewidth and long relaxation time in liquid solution [17,18]. $T_{2}$ has been measured to be $80 \mu \mathrm{s}$ at room temperature, rising to $240 \mu \mathrm{s}$ at $170 \mathrm{~K}$ [16].

The production and subsequent purification of $i-\mathrm{NC}_{60}$ are described elsewhere [19]. High-purity $i-\mathrm{NC}_{60}$ powder was dissolved in $\mathrm{CS}_{2}$ to a final concentration of $10^{15} / \mathrm{cm}^{3}$, freeze pumped to remove oxygen, and finally sealed in a quartz EPR tube. Samples were $0.7-1.4 \mathrm{~cm}$ long and contained approximately $5 \times 10^{13} i-\mathrm{NC}_{60}$ molecules. Pulsed EPR measurements were made at $190 \mathrm{~K}$ using an X-band Bruker Elexsys580e spectrometer, equipped with a nitrogen-flow cryostat.

$i-\mathrm{NC}_{60}$ has electron spin $S=3 / 2$ coupled to the ${ }^{14} \mathrm{~N}$ nuclear spin $I=1$. The EPR spectrum consists of three 
lines centered at electron $g$ factor $g=2.003$ and split by a ${ }^{14} \mathrm{~N}$ isotropic hyperfine interaction $a=0.56 \mathrm{mT}$ in $\mathrm{CS}_{2}$ [20]. Most of the pulsed EPR experiments discussed below were performed using selective pulses on the central hyperfine line in the EPR triplet, corresponding to ${ }^{14} \mathrm{~N}$ nuclear spin projection $M_{I}=0$, for which we can use a vector representation in visualizing the evolution under $\mathrm{rf}$ pulses $[8,15]$. The ESEEM experiment was performed on the $M_{I}=-1$ hyperfine line, for which a full spin density matrix treatment is necessary [16].

In order to measure the quality of an operation independently of the starting state, we define the fidelity, $\mathcal{F}$, which compares the operator for the actual rotation with that of the ideal rotation. The fidelity

$$
\mathcal{F}=\frac{1}{2} \operatorname{Tr}\left(A B^{-1}\right)
$$

takes a value between 0 and 1 , depending on how well the composite rotation $B$ approximates the ideal rotation $A$ (where $A$ and $B$ are unitary matrix operators).

A general rotation of desired angle $\theta$ with systematic error $\epsilon$, about an in-plane axis $\phi$, is given by

$$
\mathcal{R}_{\phi}[\theta(1+\epsilon)]=e^{i\left[\sigma_{x} \cos (\phi)+\sigma_{y} \sin (\phi)\right] \theta(1+\epsilon) / 2},
$$

where $\sigma_{x}$ and $\sigma_{y}$ represent the Pauli spin operators.

The BB1 corrective sequence has the form

$$
\begin{aligned}
\mathcal{R}_{0}[\theta(1+\epsilon)] \mathcal{R}_{\phi_{1}}[\pi(1+\epsilon)] \mathcal{R}_{\phi_{2}} & {[2 \pi(1+\epsilon)] } \\
& \times \mathcal{R}_{\phi_{1}}[\pi(1+\epsilon)] .
\end{aligned}
$$

The fidelity of this composite pulse can then be expanded in $\epsilon$; all orders of the expansion up to and including coefficients of $\epsilon^{5}$ equal zero for

$$
\phi_{1}=\arccos \left(-\frac{\theta}{4 \pi}\right), \quad \phi_{2}=3 \phi_{1} .
$$

For example, to achieve high-fidelity $\pi$ rotations, we choose $\phi_{1}=0.580 \pi\left(104.5^{\circ}\right)$ and $\phi_{2}=1.741 \pi\left(313.4^{\circ}\right)$. Setting phases accurately is more difficult in EPR than in NMR, owing to the higher frequency; the fidelity of a BB1 $\pi$ pulse depends on small imperfections in the pulse phases, $\delta \phi_{1}$ and $\delta \phi_{2}$, approximately as

$$
\begin{gathered}
1-\left(0.75 \delta \phi_{1}^{2}-1.125 \delta \phi_{1} \delta \phi_{2}+0.5 \delta \phi_{2}^{2}\right) \epsilon^{2} \pi^{2}, \\
-\left(0.121 \delta \phi_{1}-0.091 \delta \phi_{2}\right) \epsilon^{4} \pi^{4}+O\left(\epsilon^{6}\right) .
\end{gathered}
$$

Qubit rotation is achieved in EPR through an onresonance microwave pulse of controlled power and duration. Rotation angle errors therefore arise from either pulse duration errors (which can be assumed uniform throughout the sample) or errors in the magnitude of the microwave field, $B_{1}$, which varies across the sample depending on the homogeneity of the EPR cavity mode. For single-molecule manipulation, there is no inhomogeneity, but limitations in the resolution of power would still lead to systematic errors.
The effect of $B_{1}$ inhomogeneity is observed in the decay of Rabi oscillations as the rf pulse duration increases (see Fig. 1, solid curve). The inhomogeneity in $B_{1}$ causes an inhomogeneity in the Rabi period, so spins in different parts of the sample gradually lose coherence under the influence of the rf pulse. By removing the error accumulated over these long rotations, BB1 composite pulses can be used to obtain Rabi oscillations that would be observed in the absence of an inhomogeneous rf field.

For every desired rotation angle there are two distinct phases which are required in the correction sequence [Eq. (3)]. In order to be able to use the same two phases over the course of the experiment, the long rf pulses were divided into separate high-fidelity $\pi$ pulses, with the remainder provided by a simple pulse of length $\theta$, where $0<$ $\theta<\pi$. The BBI-Rabi oscillations shown by the dashed curve in Fig. 1 were obtained with the pulse sequence

$$
\begin{aligned}
\mathcal{R}_{0}[\theta]-\left(\mathcal{R}_{0}[\pi]-\mathcal{R}_{0.58 \pi}[\pi]\right. & -\mathcal{R}_{1.74 \pi}[2 \pi] \\
& \left.-\mathcal{R}_{0.58 \pi}[\pi]\right)_{n},
\end{aligned}
$$

and demonstrate that this sequence does not accumulate errors arising from a $B_{1}$ inhomogeneity. Since $T_{2} \approx$ $200 \mu \mathrm{s}$, which is long compared with one Rabi time period, electron spin decoherence is small on the time scale shown in Fig. 1.

In a recently reported mechanism for ESEEM, the frequency content of the echo modulation was found to have a clear dependence on the fidelity of the echo-refocusing pulse [16]. The echo decay is modulated by two frequency components, $\delta$ and $2 \delta$, whose relative amplitudes are a function of the rotation angle of the refocusing pulse. For a $\pi(1+\epsilon)=\pi+\theta_{\epsilon}$ refocusing pulse, where $\theta_{\epsilon}$ represents the (small) absolute error in the refocusing pulse, the relative magnitude of the two components is given by

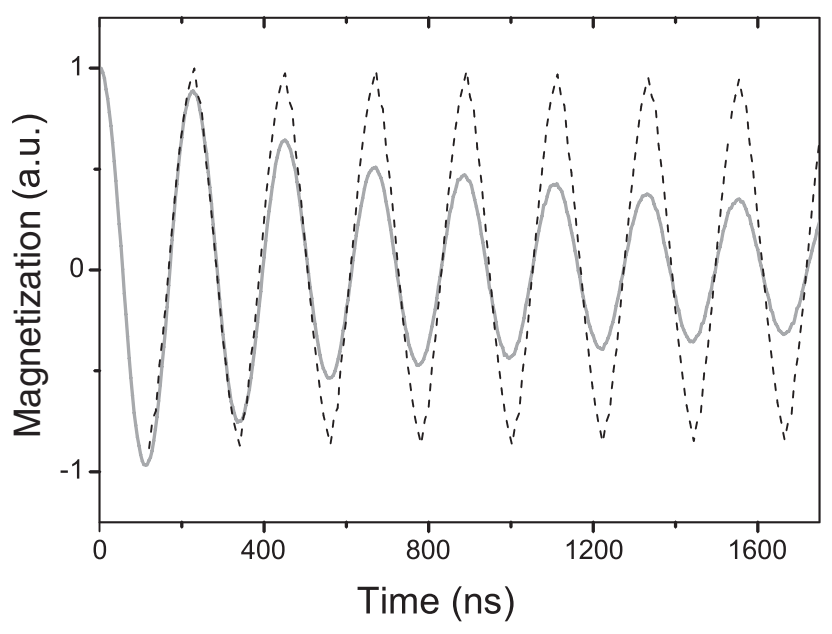

FIG. 1. Rabi oscillations for $i-\mathrm{NC}_{60}$ in $\mathrm{CS}_{2}$ at $190 \mathrm{~K}$ (solid curve). BB1-Rabi oscillations exploiting BB1 composite pulses to remove the decay caused by pulsed field inhomogeneity (dashed curve). 


$$
\frac{F(\delta)}{F(2 \delta)}=2 \theta_{\epsilon}^{2}
$$

However, for a refocusing pulse of twice the magic angle [9],

$$
\theta=2 \cos ^{-1}(\sqrt{1 / 3})+\theta_{\epsilon},
$$

the $\delta$ component dominates

$$
\frac{F(\delta)}{F(2 \delta)}=\frac{\sqrt{2}}{\theta_{\epsilon}}
$$

Figure 2 compares the Fourier transform of the echo decay using either simple or BB1 composite pulses, for nominal refocusing pulses $\theta=\pi$ and $\theta=0.608 \pi$. For the latter rotation angle, phases $\phi_{1}=0.549 \pi$ and $\phi_{2}=1.646 \pi$ were used in the BB1 pulse. When the error-compensated pulse is applied, the secondary frequency component is removed in each case, further demonstrating the ability of this composite pulse to correct for systematic errors in rotation angle. It is worth noting that in these ESEEM experiments, the BB1 "refocusing" pulses operate on spins which are dispersed in the rotating $x-y$ plane. This illustrates the effectiveness of the BB1 composite pulse over a range of initial states.

Rotation angle errors can be measured by comparing the rates of echo decay in two multipulse sequences: CarrPurcell (CP) and Carr-Purcell-Meiboom-Gill (CPMG) $[8,9]$. The echo decay in CPMG shows no sensitivity to rotation angle errors (after every even numbered cycle) and is dictated purely by decoherence. Echo magnitudes in $\mathrm{CP}$ show a cumulative sensitivity to errors in the refocusing $\pi$ pulses and hence decay faster than in CPMG, as shown in Fig. 3. By performing a comparison as described in Ref. [8], we estimated that the refocusing pulses had a standard error of $0.1 \pi\left(18^{\circ}\right)$, equivalent to a fidelity of $\mathcal{F}=0.988$.

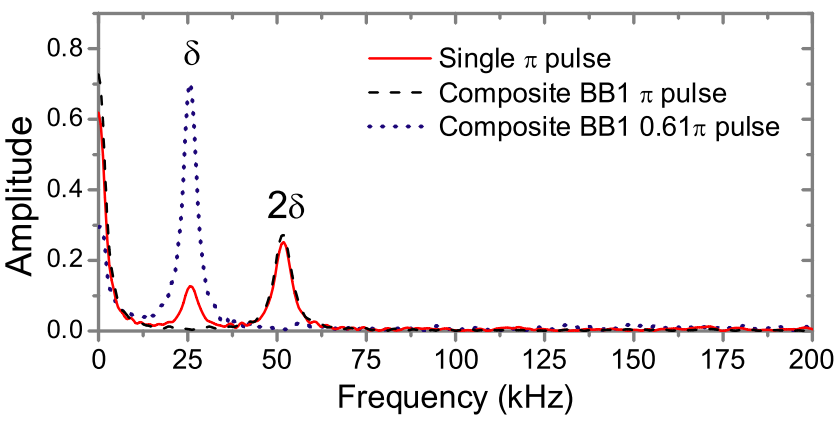

FIG. 2 (color online). The Fourier transform of ESEEM in $i-\mathrm{NC}_{60}$ shows two frequency components of 26 and $52 \mathrm{kHz}$. For simple $\pi$ refocusing pulses, the $26 \mathrm{kHz}$ component is attributed to pulse error (solid line) and is removed when a BB1 composite $\pi$ pulse is used (dashed line). For a refocusing pulse of approximately $0.61 \pi$, the $52 \mathrm{kHz}$ component, which would be present due to pulse error, can also be removed by using a BB1 composite $0.61 \pi$ pulse (dotted line).
By replacing the $\pi$ pulses in CP with BB1 composite $\pi$ pulses, we are able to make an estimate of the residual rotation angle error in the high-fidelity rotation. The error accumulated in the $\mathrm{CP}$ sequence is dependent on the number of cycles applied, and hence the sensitivity of this technique is limited only by the maximum number of pulses that can be applied in one experiment (with the spectrometer used for these experiments, this is currently 32, which implies fewer than $8 \mathrm{BB} 1$ composite rotations). From the decay of the BB1 corrected CP sequence, we are able to conclude that the rotation angle error is at most $0.01 \pi\left(2^{\circ}\right)$, corresponding to a fidelity of $\mathcal{F}>0.9993$. The phases used in this BB 1 experiment were measured using the SPAM sequence [8] and found to be $\phi_{1}=(0.587 \pm$ $0.008) \pi$ and $\phi_{2}=(1.742 \pm 0.011) \pi$. Using Eq. (5) these imply an expected fidelity of $\mathcal{F}=0.9999$.

Error correcting codes will be crucial to the operation of a quantum computer [21], compensating for both decoherence errors and the gate operation errors described here. The threshold of error probability for these codes varies as a function of the overhead, but generally lies between $10^{-3}$ and $10^{-4}$, or a fidelity greater than 0.999 [22]. Despite the inherent $10 \%$ systematic error, the composite pulses described here are capable of producing operations which meet this threshold fidelity. However, errors in pulse phase cause the fidelity to fall short of the theoretical optimum of $1-10^{-6}$. In addition to the BB1 composite pulse described here, longer composite pulses have been proposed which can arbitrarily reduce the sensitivity to systematic error [13]. In order for these higher-order composite pulses to be effective in pulsed EPR, greater phase control is required.

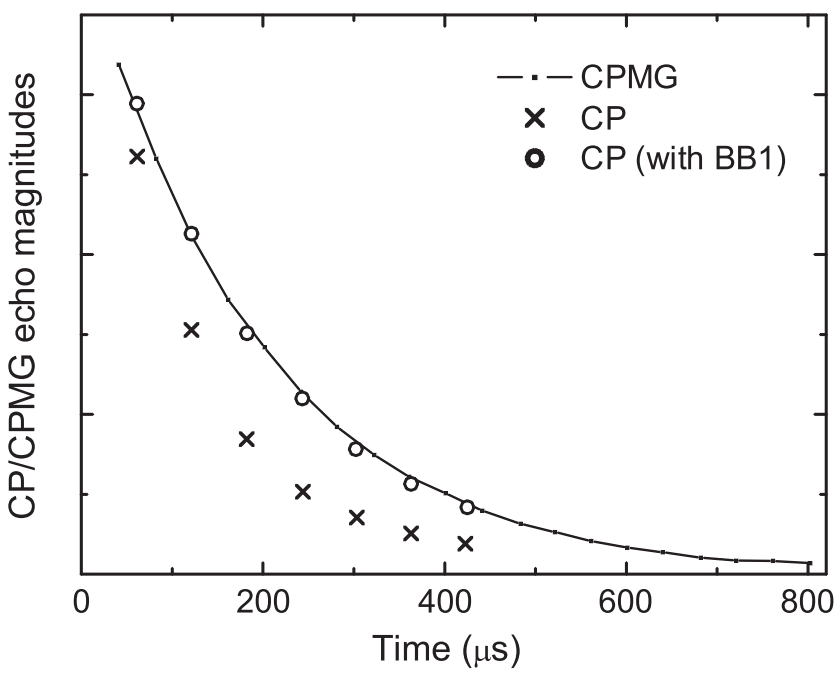

FIG. 3. Comparison of the echo signal decays in the reference CPMG sequence (dots) with that of the error-sensitive $\mathrm{CP}$ (crosses) sequence provides a measure of rotation angle errors. $\mathrm{CP}$ echoes generated with simple $\pi$ pulses decay more rapidly owing to rotation angle errors. When the simple $\pi$ pulses are replaced with BB1 composite $\pi$ pulses (open circles), the decay rate is very close to that in CPMG. 
An analysis of random errors present would complement the current work in determining where the compromise between lengthy pulse sequences and error reduction lies.

We would like to thank Wolfgang Harneit's group at the Hahn-Meitner Institute for providing nitrogen-doped fullerenes, and John Dennis (QMUL), Martin Austwick, and Gavin Morley for the purification of $i-\mathrm{NC}_{60}$. We also thank Jonathan Jones for stimulating and valuable discussions, and the Oxford-Princeton Link fund for support on this project. This research is part of the QIP IRC www. qipirc.org (GR/S82176/01). G. A. D. B. thanks EPSRC for financial support (GR/S15808/01). A. A. is supported by the Royal Society. Work at Princeton was supported by the NSF International Office through the Princeton MRSEC Grant No. DMR-0213706 and by the ARO and ARDA under Contract No. DAAD19-02-1-0040.

*Electronic address: john.morton@materials.ox.ac.uk

[1] M. A. Nielsen and I.L. Chuang, Quantum Computation and Quantum Information (Cambridge University Press, Cambridge, 2000).

[2] D. G. Cory, A. F. Fahmy, and T. F. Havel, Proc. Natl. Acad. Sci. U.S.A. 94, 1634 (1997).

[3] W. S. Warren, Science 277, 1688 (1997).

[4] G. Burkard, H. A. Engel, and D. Loss, Fortschr. Phys. 48, 965 (2000).

[5] W. Harneit, Phys. Rev. A 65, 032322 (2002).

[6] A. Ardavan et al., Phil. Trans. R. Soc. A 361, 1473 (2003).

[7] S. A. Lyon, cond-mat/0301581.
[8] J. J. L. Morton, A. M. Tyryshkin, A. Ardavan, K. Porfyrakis, S. A. Lyon, and G. A. D. Briggs, Phys. Rev. A 71, 012332 (2005).

[9] R. Freeman, Spin Choreography: Basic Steps in High Resolution NMR (Oxford University Press, New York, 1997).

[10] M. H. Levitt, Prog. Nucl. Magn. Reson. Spectrosc. 18, 61 (1986).

[11] S. Wimperis, J. Magn. Reson., Ser. A 109, 221 (1994).

[12] H. K. Cummins, G. Llewellyn, and J.A. Jones, Phys. Rev. A 67, 042308 (2003).

[13] K. R. Brown, A. W. Harrow, and I.L. Chuang, Phys. Rev. A 70, 052318 (2004).

[14] E. Collin, G. Ithier, A. Aassime, P. Joyez, D. Vion, and D. Esteve, Phys. Rev. Lett. 93, 157005 (2004).

[15] A. Schweiger and G. Jeschke, Principles of Pulse Electron Paramagnetic Resonance (Oxford University Press, New York, 2001).

[16] J. J. L. Morton, A. M. Tyryshkin, A. Ardavan, K. Porfyrakis, S.A. Lyon, and G. A. D. Briggs, J. Chem. Phys. 122, 174504 (2005).

[17] E. Dietel, A. Hirsch, B. Pietzak, M. Waiblinger, K. Lips, A. Weidinger, A. Gruss, and K. P. Dinse, J. Am. Chem. Soc. 121, 2432 (1999).

[18] C. Knapp, K. P. Dinse, B. Pietzak, M. Waiblinger, and A. Weidinger, Chem. Phys. Lett. 272, 433 (1997).

[19] M. Kanai, K. Porfyrakis, G. A. D. Briggs, and T. J.S. Dennis, Chem. Commun. (Cambridge) 2, 210 (2004).

[20] T. Almeida-Murphy, T. Pawlik, A. Weidinger, M. Hohne, R. Alcala, and J. M. Spaeth, Phys. Rev. Lett. 77, 1075 (1996).

[21] A. M. Steane, Phys. Rev. A 68, 042322 (2003).

[22] Y.C. Cheng and R. J. Silbey, quant-ph/0412168. 